\title{
Arthroskopie am oberen Sprunggelenk: Möglichkeiten und Grenzen
}

\author{
Atesch Ateschrang, Steffen Schröter, Ulrich Stöckle
}

\section{Zusammenfassung}

Die Arthroskopie des oberen Sprunggelenks hat sich in den letzten Jahren sowohl in der Feindiagnostik als auch zur Therapie etabliert und ist nicht mehr wegzudenken. Die Arthroskopie ermöglicht durch funktionelle Diagnostik intraoperativ die Evaluierung von Instabilitäten, Objektivierung bzw. Ausschluss des Impingements sowie das Knorpelstaging zur stadiengerechten Knorpelrekonstruktion von Knorpelschäden bzw. osteochondraler Läsionen. Des Weiteren ist die Bergung freier Gelenkkörper sowohl zentral als auch im Bereich des Außen- und Innenknöchels möglich. Diese und knorpeltherapeutische Interventionen können teilweise oder komplett arthroskopisch erfolgen. Dieser Artikel soll eine aktuelle Übersicht geben.

\begin{abstract}
Arthroscopy of the ankle has become accepted for diagnostics and therapeutical interventions and is an indispensible tool for clinical routine work. Arthroscopic intervention of the ankle allows precise intraoperative functional diagnostics for evaluating instability, impingement, as well as cartilage staging for stage dependant cartilage repair. Loose joint bodies in the central compartment as well as underneath the medial and lateral malleolus are removable. These procedures can be done arthroscopically or open. This article gives a current overview.
\end{abstract}

\section{Einleitung}

Die Arthroskopie (ASK) des oberen Sprunggelenks (OSG) ist eine sichere und effektive Methode zur Beurteilung der Gelenkbinnenstrukturen sowie zur Prüfung der ligamentären Stabilität. Die Weiterentwicklungen der arthroskopischen Chirurgie in den vergangenen 510 Jahren führten zu einer enormen $\mathrm{Er}$ weiterung der Behandlungsmöglichkeiten am oberen und auch am unteren Sprunggelenk, die noch vor 10-15 Jahren der offenen Chirurgie vorbehalten waren. Arthroskopisch können vielfältige pathologische Befunde identifiziert und behandelt werden, wie das knöcherne und Weichteilimpingement, Knorpelschäden, osteochondrale Läsionen, Instabilitäten, freie Gelenkkörper, Adhä-

OP-JOURNAL 2014; 30: 112-118

(c) Georg Thieme Verlag KG Stuttgart · New York DOI http://dx.doi.org/10.1055/s-0034-1383266

sionen mit Bewegungseinschränkung und das Gelenkempyem. Überschneidungen zu offenen Verfahren bestehen nach wie vor, wobei die arthroskopischen Techniken nicht nur als konkurrierende, sondern ergänzende Verfahren angesehen werden sollten, wie bspw. bei unspezifischer bzw. nicht abgrenzbarer Beschwerdesymptomatik. Gerade für solche Fälle können Gelenkbinnenprobleme ggf. ausgeschlossen oder bestätigt werden, um bedarfsweise gleichzeitig vorliegende Peronäalsehnen-, Rückoder Vorfußpathologien zu behandeln. Mit den nachfolgenden Ausführungen sollen die klinisch relevanten Behandlungsmöglichkeiten, aber auch Grenzen der Arthroskopie zusammengefasst werden.

\section{Diagnostik}

Die fundierte Indikationsstellung zur OSG-Arthroskopie umfasst eine detaillierte klinische Untersuchung sowie
Röntgenaufnahmen in 2 Ebenen. Schrägaufnahmen können jedoch durchaus sinnvoll sein. Aufgrund der topografisch engen Beziehung zum unteren Sprunggelenk, aber auch zu der Peronäalsehnenloge und zum Tarsaltunnel sollte vor operativen Interventionen die Indikation zur Kernspintomografie (MRT) eher großzügig gestellt werden. Bei Folgezuständen nach Frakturen mit einliegenden Implantaten erlaubt die MRT nicht immer den gewünschten Informationsgewinn. Für solche Fälle ist die Computertomografie (CT) sinnvoll.

Topografisch sollten die Pathologien in ventrale und dorsale OSG-Pathologien unterschieden werden, da die ventrale arthroskopische Intervention einfacher und die neurovaskuläre Komplikationswahrscheinlichkeit deutlich niedriger ist als die dorsalen Interventionen. An dieser Stelle ist zu erwähnen, dass das chirurgische Vorgehen sich immer am klinischen Beschwerdebild orientieren sollte und keine MR- bzw. Röntgenbefunde isoliert operativ angegangen werden sollten.

Basis jeder Behandlung ist die gründliche klinische Untersuchung und Röntgenaufnahmen in 2 Ebenen vor Durchführung einer MRT.

\section{Impingement}

Das Impingement kann einerseits nach der Lokalisation (ventral, ventromedial, ventrolateral, dorsal, dorsomedial und dorsolateral) und andererseits nach den auslösenden Strukturen (Synovia, postraumatische Narben, Kapselbandstrukturen, knöchern durch Osteophyten oder freie bzw. adhärente Gelenkkörper) eingeteilt werden [1]. Das ventrale Weichteilimpingement ist häufig eine posttraumatische Folge nach Distorsionsereignissen. Durch die Distorsion oder rezidivierende Mikrotraumen kann es zu Kapselband- sowie Synovialrupturen kommen. Die verletzten Bandstrukturen 


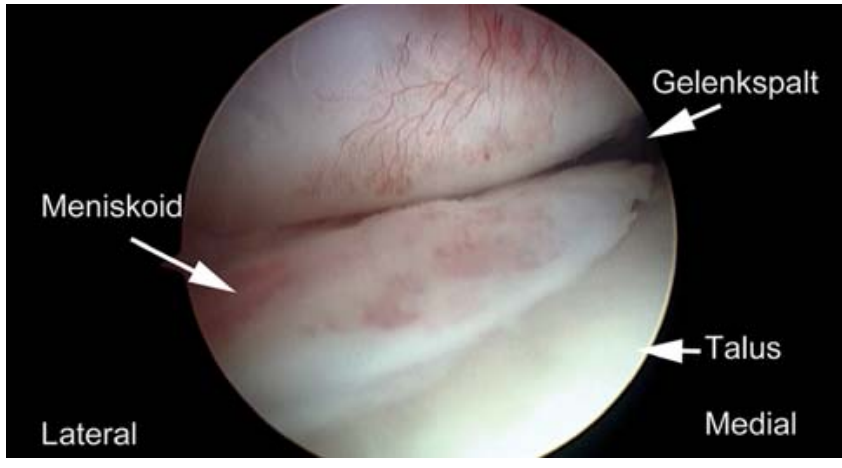

Abb. 1 Intraoperativer Befund eines Meniskoids des rechten OSG mit Blick über das anterolaterale Standardportal.

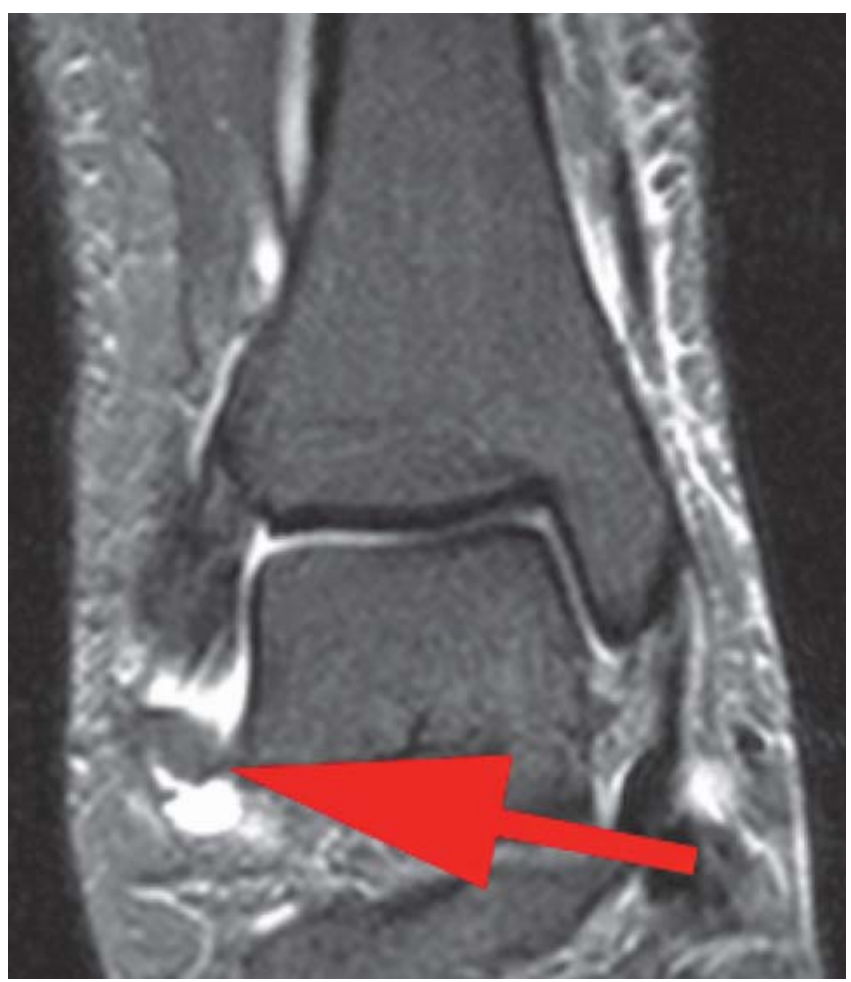

Abb. 3 Beispiel eines knöchernen fibularen Ausrisses des Lig. fibulotalare anterius (LFTA).

können in den anterolateralen oder anteromedialen Recessus einklemmen oder durch Bridenbildung dauerhafte Gelenkbeschwerden hervorrufen. Durch zusätzliche Kapsel- und Synovialrupturen kann diese posttraumatische Bridenbildung bis in den anterioren zentralen Gelenkrecessus reichen, wobei in diesem Zusammenhang die Bezeichnung des „Meniskoids“ häufig Anwendung findet. Diese Veränderungen können zu persistierenden Beschwerden des OSG führen und in einzelnen Fällen sogar Gelenkblockaden hervorrufen (Abb. 1 und 2). Dabei sind die konventionell-radiologische Bildgebung sowie das MRT nicht immer wegweisend.

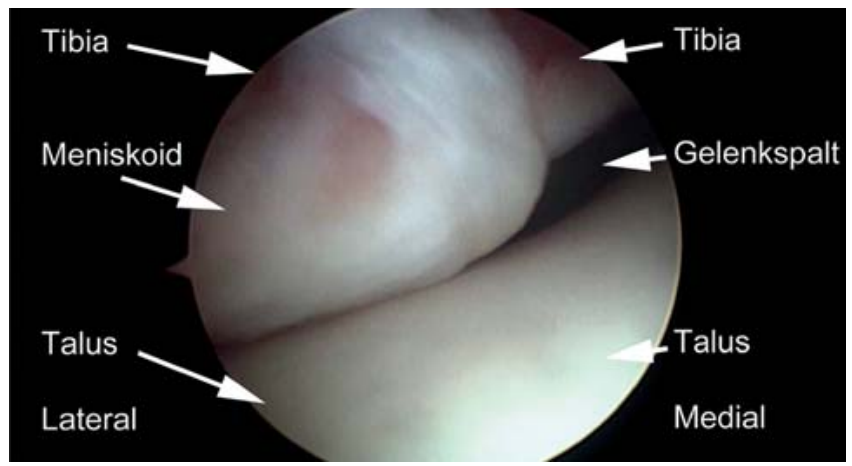

Abb. 2 Weiteres Beispiel eines Meniskoids des rechten OSG mit Blick über das anterolaterale Standardportal.

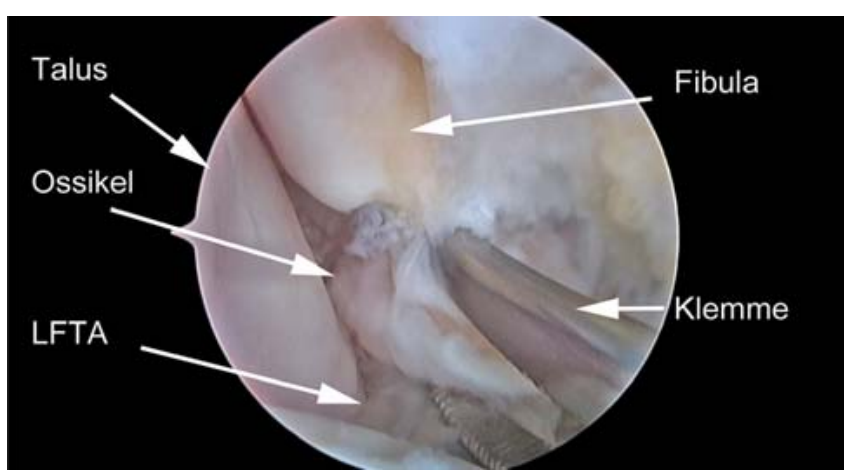

Abb.4 Intraoperatives Beispiel eines Außenknöchelossikels mit isolierten lokalen Beschwerden.
Sprunggelenk. Allerdings sollte dabei eine höhere neurovaskuläre Komplikationsrate Beachtung finden. Nickisch et al. fanden nach 189 dorsalen arthroskopischen Interventionen in 8,5\% Taubheiten der Ferse, N.-suralis-Läsionen und symptomatische Achillessehnenverdickungen [2].

Trotz unauffälligem MRT sollte die Arthroskopie insbesondere bei persistierenden Beschwerden erfolgen, da die Sensitivität nicht sehr hoch ist.

Die Wertigkeit der MRT zur Impingementdiagnostik wird kontrovers diskutiert. Auch nach KM-Injektion können falsch negative Befunde vorliegen. Die Hauptursache hierzu liegt in der Lagerung der Sprunggelenke in Plantarflexion und nicht in Neutralstellung.

Die dorsale Impingementpathologie kann, neben synovialen Strukturen bzw. Vernarbungen, ein symptomatisches Os trigonum, fehlverheilte knöcherne Absprengungen oder osteophytäre Anbauten enthalten. Diese können analog zum ventralen Vorgehen von dorsal in Bauchlage arthroskopisch adressiert werden oder alternativ bei hängendem Bein mit einem zirkumferent frei zugänglichen

\section{Bänder}

Insbesondere für die Beurteilung der Bandstrukturen ist die Neutralstellung wünschenswert. Lässt sich trotz unauffälliger Bildgebung (Röntgen und MRT) und erfolgter konservativer Therapie mit Physiotherapie, Propiozeptionstraining sowie physikalischen Maßnahmen keine Beschwerdelinderung erzielen, sollte daher die Indikation zur Arthroskopie großzügig gestellt werden. Kleine knöcherne ligamentäre Ausrissläsionen am Innen- und Außenknöchel (Abb. 3 und 4) sollten von rezidivierenden Mikrotraumatisierungen differenziert werden. 


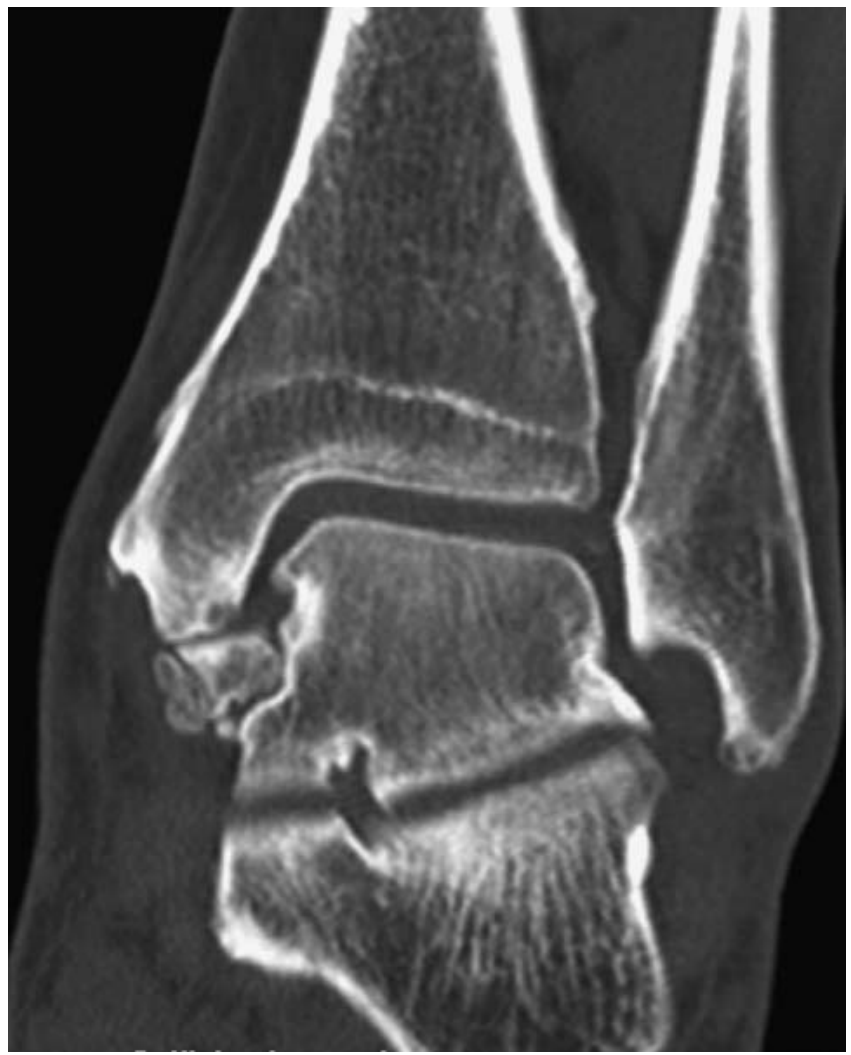

Abb.5 Beispiel eines professionellen Fußballspielers mit rezidivierenden Mikrotraumatisierungen (Supinationstraumen) und konsekutiver Ossikelbildung und Kalzifikationen des Lig. deltoideum.

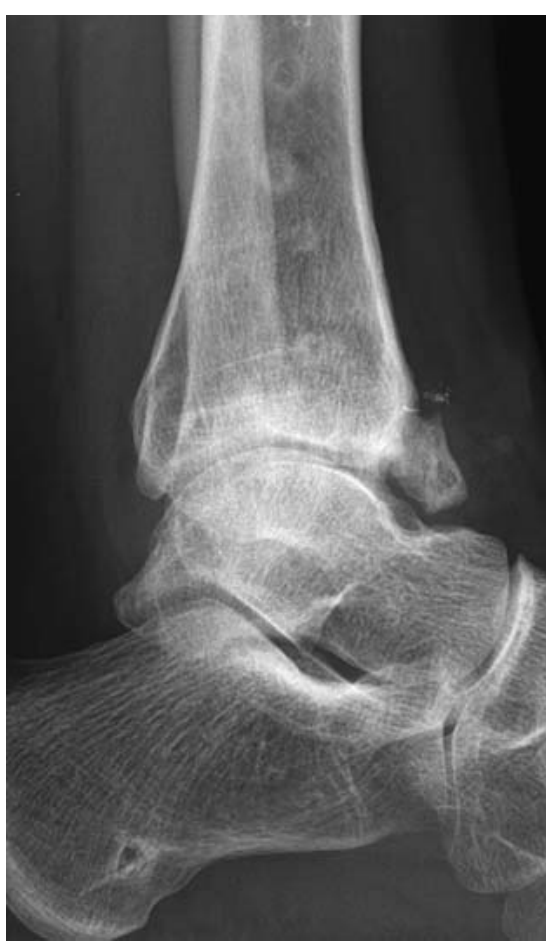

Abb. 7 Ausgeprägter tibialer Osteophyt bei mäßiger posttraumatischer OSG-Arthrose nach Pilonfraktur einer 23-jährigen Patientin.

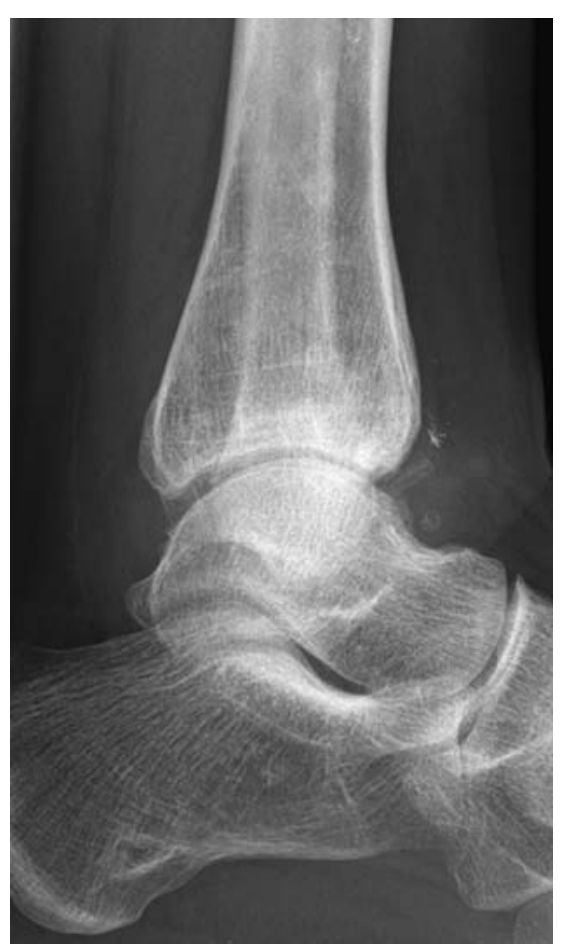

Abb. 8 Postoperative Situation nach arthroskopischer Abtragung des tibialen Osteophyten.

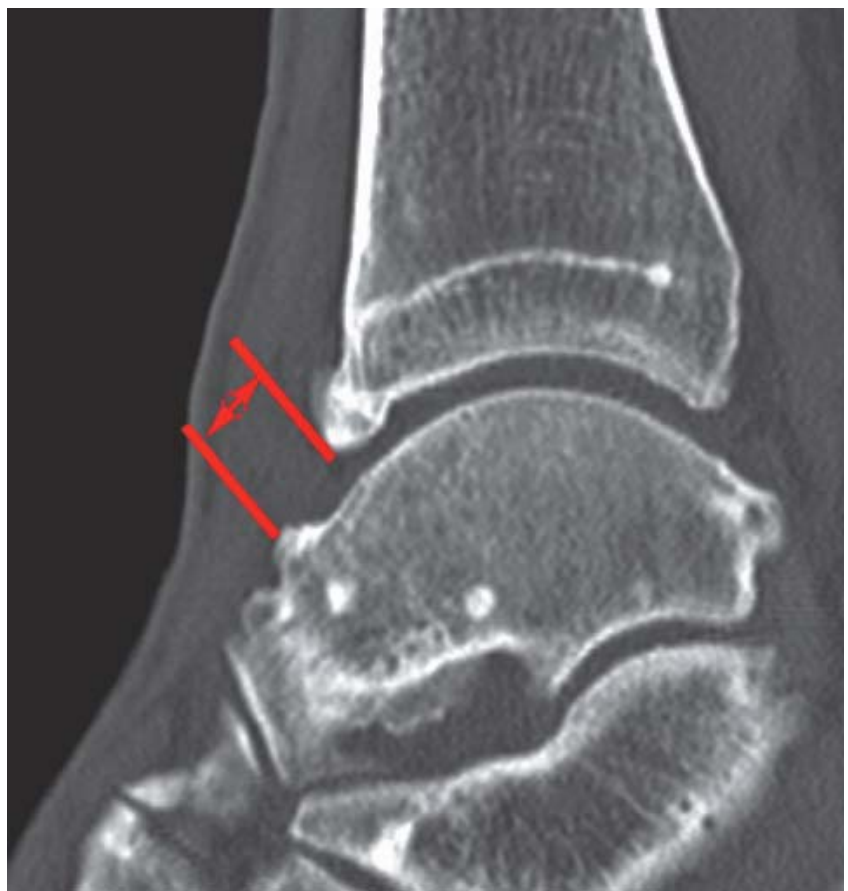

Abb. 6 Die Impingementklassifikation besteht aus Grad I-III, wobei die Distanz des talaren und tibialen Osteophyten ausgemessen wird. Grad I: $\geq 10$ mm, Grad II: 5-10 mm, Grad III: $\leq 5 \mathrm{~mm}$.

\section{Knöcherne Läsionen und Arthrose}

Diese rezidivierenden Traumata (Eversions- und Supinationsmechanismus) können zu osteophytären Anbauten und Ossikelbildung bzw. zu ligamentären Ossifikationen führen (Abb. 5). Die Klassifizierung ist nach Scranton und McDermot möglich oder alternativ nach Vorschlag der Autoren (Abb.6). Diese Gelenkbinnenpathologien können arthroskopisch sehr gut adressiert und komplett ausgeräumt bzw. Osteophyten mit unterschiedlich dimensionierten Fräsen entfernt werden (Abb. 7 und 8).

Die gelenkerhaltenden Behandlungsmöglichkeiten der OSG-Arthrose umfassen, neben der Einlagenversorgung, die Abrollsohle ggf. auch in Kombination mit orthopädischen Stiefeln sowie die Schmerztherapie. Im eigenen Vorgehen kann die arthroskopische Revision mit Synovektomie bei konventionell-radiologisch einsehbarem Gelenkspalt v.a. bei osteophytär bedingtem knöchernem Impingement Beschwerdelinderung erzielen. Klinisch muss in diesen Fällen der Arthroseschmerz vom impingementbedingten Schmerz differenziert werden. Insbesondere der ventrale OSGSchmerz, der bei endgradiger Dorsalextension auftritt, kann durch die Entfernung der miteinander in Konflikt geratenden Osteophyten (ventraler tibialer 


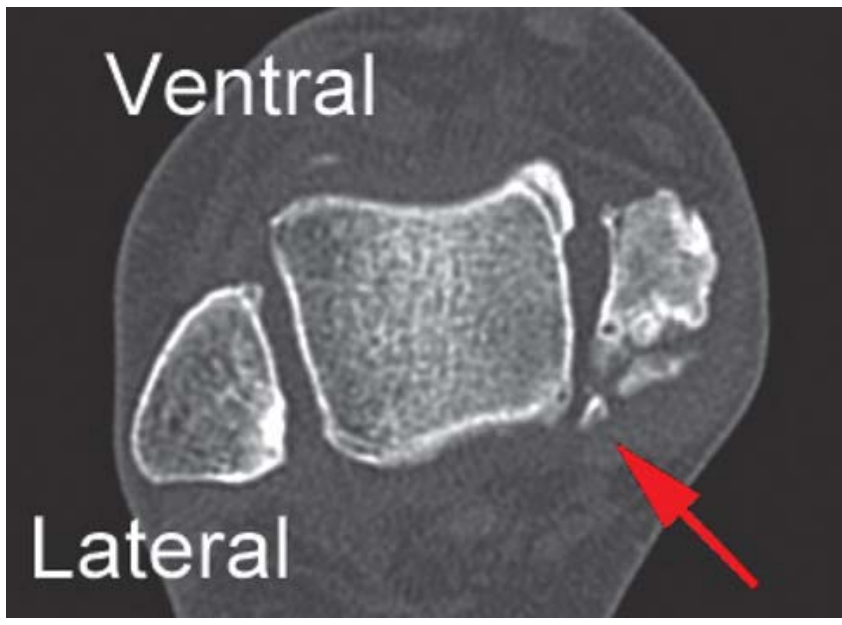

Abb.9 Posttraumatisches Impingement des OSG im Bereich des Innenknöchels nach Fraktur.

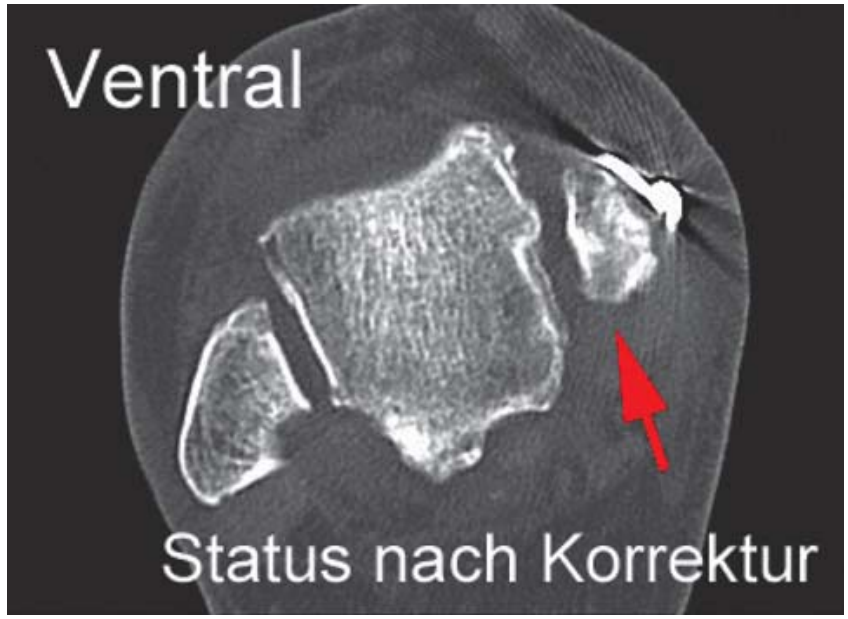

Abb. 10 Postoperatives Ergebnis nach Korrekturosteotomie des Innenknöchels mit Entfernung der Frakturfragmente und Stabilisierung mittels Zuggurtung. und talarer Osteophyt) deutlich gelindert werden [3]. Grundsätzlich empfehlen wir für diese Fälle großzügig die Durchführung einer CT. Die CT ist sehr hilfreich zur Beurteilung, ob und wo ggf. ein Impingement des OSG vorliegt, sodass für geeignete Fälle ggf. auch die dorsale arthroskopische Intervention erfolgen könnte.

Zu Planung der Osteophytenentfernung empfiehlt sich besonders eine $\mathrm{CT}$, um möglichst gezielt operativ vorzugehen.

\section{Knöcherne Beinachse}

Folgezustände nach Frakturen sollten sehr sorgfältig analysiert werden, um posttraumatische Achs- und Rotationsabweichungen zu identifizieren, da diese zwar zu einem Impingement führen, aber nur durch eine Korrekturosteotomie beherrscht werden können (Abb. 9 und 10). In solchen Fällen sollte aber zusätzlich eine Arthroskopie erfolgen, um koinzidente intraartikuläre Pathologien auszuschließen. Falls durch die Arthroskopie intraoperativ eine zu starke Schwellung auftritt, kann ggf. ein zweizeitiges Vorgehen für die offene Operation nötig werden, um Wundheilungsstörungen zu vermeiden.

\section{Knorpelschäden und osteochondrale Läsionen}

Symptomatische Knorpelschäden sollten mittels MRT sorgfältig diagnostisch analysiert werden, um die Größe der Läsion und deren Tiefenausdehnung für eine optimale Therapieplanung im Vorfeld zu wissen. Die Klassifikation des Knorpelschadens erfolgt nach ICRS-Kriterien
(Grad 0-IV). Entscheidend ist hierbei die subchondrale Knochenqualität. Zeigt sich keine Pathologie des Knochens, so kann man sich isoliert auf den Knorpelschaden konzentrieren. Läsionen Grad I und II bedürfen lediglich einer Knorpelglättung und einer kritischen Ursachenanalyse, wie bspw. osteophytär bedingter Knorpelabrieb, freie Gelenkkörper, Weichteilimpingement oder Instabilitäten, die dann adressiert werden müssen, da ansonsten ein Fortschreiten des Knorpelabriebs die Folge ist. Dies gilt grundsätzlich für jeden Knorpelschaden, unabhängig von Größe und Klassifikation.

\section{Ortständige Reparatur bzw. Mikrofrakturierung}

Bei Knorpelschaden Grad III und IV kann ohne subchondrale Knochenläsion die Mikrofrakturierung (MF) bei einer Größenausdehnung von bis zu $1,5 \mathrm{~cm}^{2}$ oder $15 \mathrm{~mm}$ im Durchmesser erfolgen [4-6]. Die Vorteile der arthroskopischen MF bedeuten niedrige Kosten sowie technisch einfache und reproduzierbare Durchführbarkeit mit geringem postoperativem Schmerzniveau. Im praktischen Vorgehen sollten die Abstände der MF (Perforationen) ca. 3-4 mm betragen (Abb. 11 und 12). Die adäquate Perforationstiefe kann entweder durch Austritt von Knochenmarksfettpfropfen oder Blutungen (bei geöffnetem Tourniquet) aus der Läsion dokumentiert werden. $\mathrm{Zu}$ beachten ist die Schaffung stabiler Knorpelränder. Die Einblutung in die Läsion führt zu einer inflammatorischen Reaktion und Einsprossung mesenchymaler Stammzellen, die sich zu chondrozytenähnlichen Zellen differenzieren. Diese bilden Kollagen Typ II in einer Pro- teoglykanmatrix 6-8 Wochen postinterventionell [4,7]. Die darauffolgende Oberflächenfibrillation, die Apoptose der chondrozytenähnlichen Zellen sowie die Depletion der Proteoglykane führt zu einem biologischen Transfer in fibrokartilaginäres Reparaturgewebe mit einer überwiegenden Expression von Kollagen-Typ-I-Fasern nach ca. 1 Jahr. Typ-IKollagenfasern haben unterschiedliche biomechanische Eigenschaften gegenüber hyalinem Knorpel, sodass degenerative Veränderungen im Langzeitverlauf auftreten können [7].

Den Autoren ist keine Langzeitstudie mit einem 10-Jahres-Follow-up zur MF am Talus bekannt. Bisher gibt es keine systematischen Erkenntnisse, welche Lokalisation am Talus besonders gut bzw. als ungünstig für die MF zu bewerten ist. Die Prognose für die MF ist größenabhängig und günstig für Läsionen, die $<15 \mathrm{~mm}^{2}$ sind. Dennoch zeigt die bisherige Datenlage insbesondere für den mittel- bis längerfristigen Verlauf eine klinische Verschlechterung nach initial guten Ergebnissen, sodass weitere hochwertige Studien notwendig sind zur Entwicklung eines Therapiealgorithmus [4].

Die Mikrofrakturierung allein ist für Knorpelschäden bis zu $1,5 \mathrm{~cm}^{2}$ ohne relevante zystische subchondrale Knochenläsionen geeignet.

\section{Knorpeltransplantation}

Für größere Läsionen ohne subchondralen Knochendefekt kommt die autologe Chondrozytentransplantation (ACT) infrage. Im Rahmen der präoperativen Diagnostik und der ASK erfolgt das Staging 


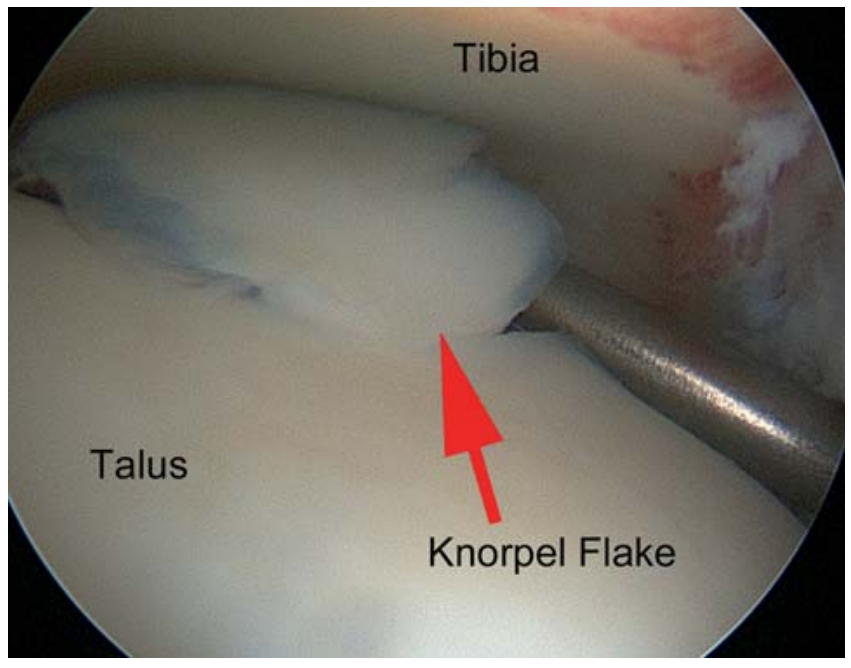

Abb. 11 Posttraumatischer instabiler Knorpel Flake nach OSG-Supinationstrauma an der lateralen Talusschulter.

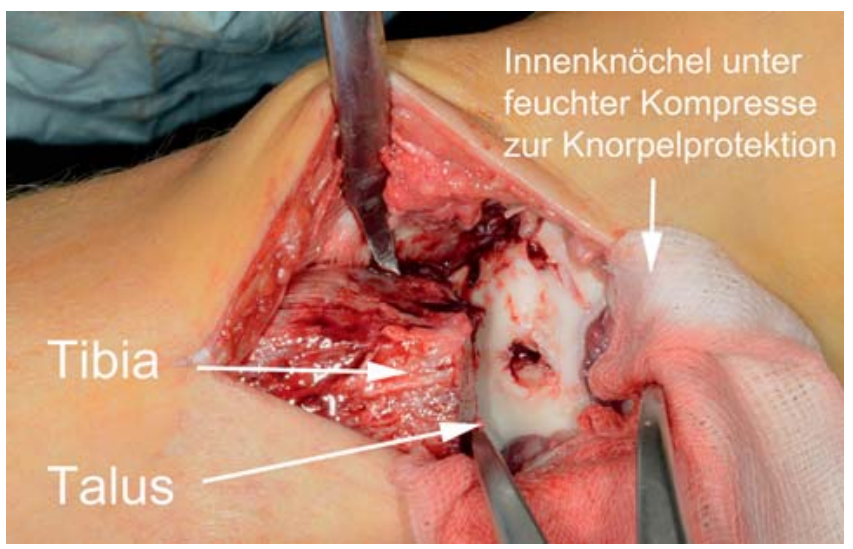

Abb. 13 Osteochondrosis dissecans des medialen Talus mit Grad-IIIKnorpelschaden (ICRS) sowie zystischem subchondralem Defekt nach Innenknöchelosteotomie.

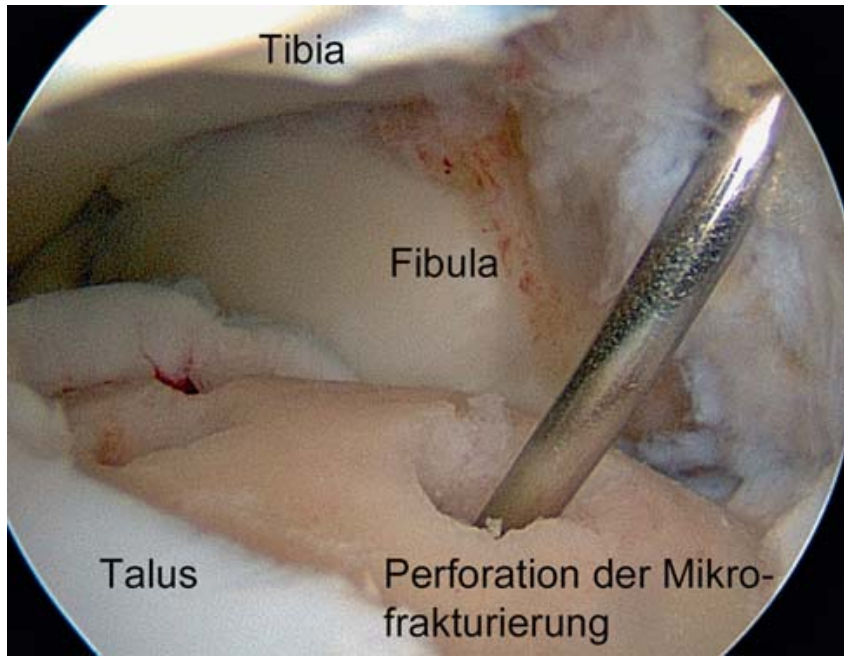

Abb. 12 Status nach Debridement und Mikrofrakturierung mit 2 Perforationen der lateralen Talusschulter.

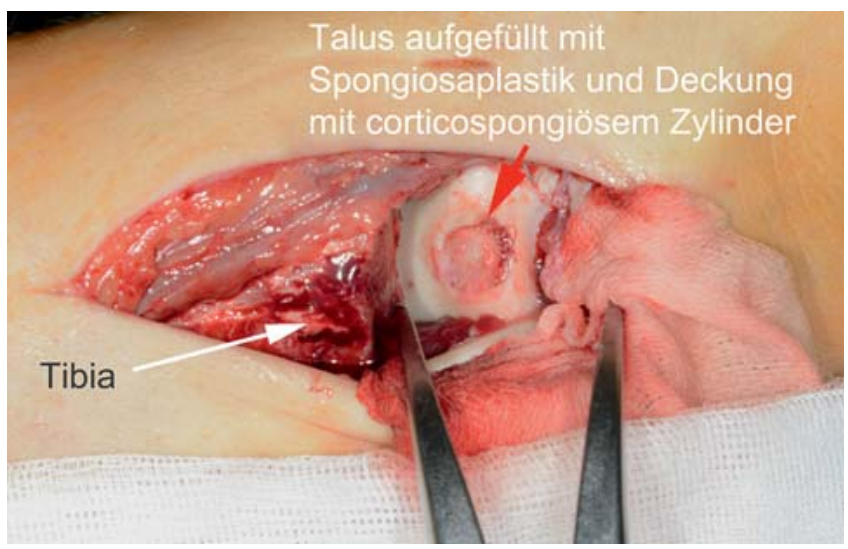

Abb. 14 Situs nach erfolgter Auffüllung der Zyste und Rekonstruktion der subchondralen Oberfläche im Bereich der Stanze mit einem kortikospongiösen Zylinder. mit Ursachenanalyse, um bei gegebener Indikation die Knorpelzellentnahme am Talushals zentral außerhalb der Belastungszone zu realisieren. Die gewonnenen Knorpelzellen werden kultiviert, um nach 3-4 Wochen arthroskopisch unter Distraktion in „Inject-Technik“ in die Knorpeldefektzone eingespritzt $\mathrm{zu}$ werden. Besteht neben dem Knorpelschaden zusätzlich ein subchondraler Knochendefekt (Osteochondrosis dissecans, kurz: OD) muss dieser adäquat rekonstruiert werden. Neben einer osteochondralen Transplantation (OCT) aus dem Kniegelenk ist ein kortikospongiöser Transfer vom Beckenkamm entweder mit einer matrixassoziierten ACT (MACT - Beispiel in Abb. 11-13) oder mit einer 3-D-Kollagenmembran (AMIC - autologe matrixinduzierte Chondrogenese) möglich. Die Datenlage für das AMIC-Verfahren ist allerdings noch sehr begrenzt.
Die OCT kann für geeignete Fälle arthroskopisch oder in offener Technik erfolgen. Historisch erfolgte die OCT für zystische Läsionen des Talus oder bei persistierenden Beschwerden nach vorangegangenen Eingriffen. Die Indikationserweiterung für die primäre OCT beinhaltet zwischenzeitlich auch nicht nur zystische Läsionen bzw. Knochendefekte, sondern insbesondere auch großflächige Knorpelschäden des Talus [4]. Vorteile der OCT sind die primäre Deckung des Defekts mit vitalem hyalinem Knorpel ohne die Notwendigkeit eines weiteren Eingriffs. Technisch bedingte Nachteile schließen die limitierte Verfügbarkeit des Spenderareals, die Entnahmemorbidität sowie die unterschiedliche anatomische Formgebung des Spender- und Empfängerareals ein. Dies betrifft sowohl den Knorpel (Schichtdicke) als auch den Knochen (Konvexität). Zusätzlich ist die schlechte Einheilung der Knorpelränder im Bereich des Spender-
Empfänger-Interfaces zu nennen. Klinisch wurden in bis zu $95 \%$ gute bis sehr gute Ergebnisse erzielt. Symptomatische Entnahmemorbiditätsraten von bis zu $4 \%$ wurden dabei ermittelt [4]. Hierfür notwendige Innenknöchelosteotomien heilten durchschnittlich innerhalb von 6 Wochen. MR-Kontrollen zeigten im Bereich der Osteotomie eine normale Signalgebung für hyalinen Knorpel in den tiefen Schichten und für die oberflächlichen $50 \%$ eine faserknorpelähnliche Signalgebung [8]. Die genauere Untersuchung der Entnahmemorbidität erfolgte in einer größeren Serie von 112 asymptomatischen Kniegelenken mit der Ermittlung des WOMAC- sowie Lysholm-Scores [9]. Der jeweils durchschnittliche WOMAC-Score betrug 5\% und der Lysholm-Score zählte 89 Punkte. Als Risikofaktor für Beschwerden konnte Übergewichtigkeit identifiziert werden, wobei überraschenderweise nicht die 


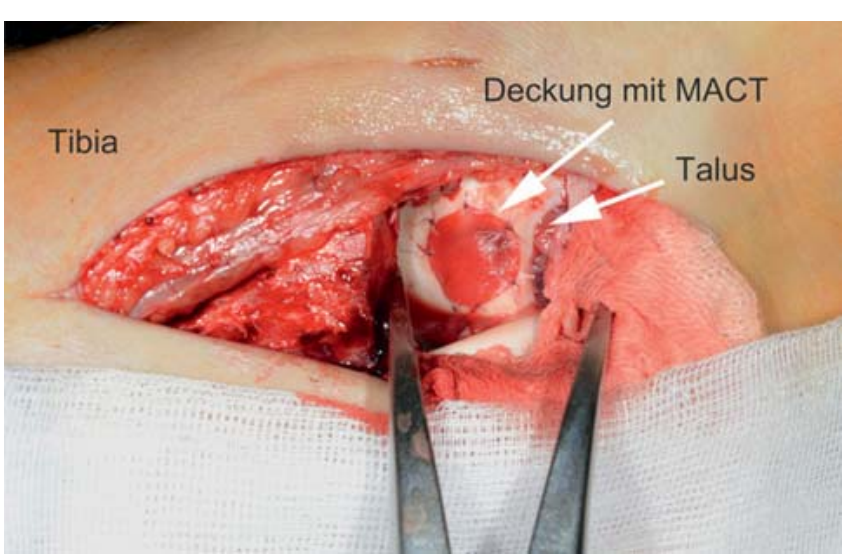

Abb. 15 Situs nach knöcherner Rekonstruktion und Débridement des Knorpelschadens sowie Deckung mit der autologen MACT $(18 \mathrm{~mm}$ im Durchmesser).

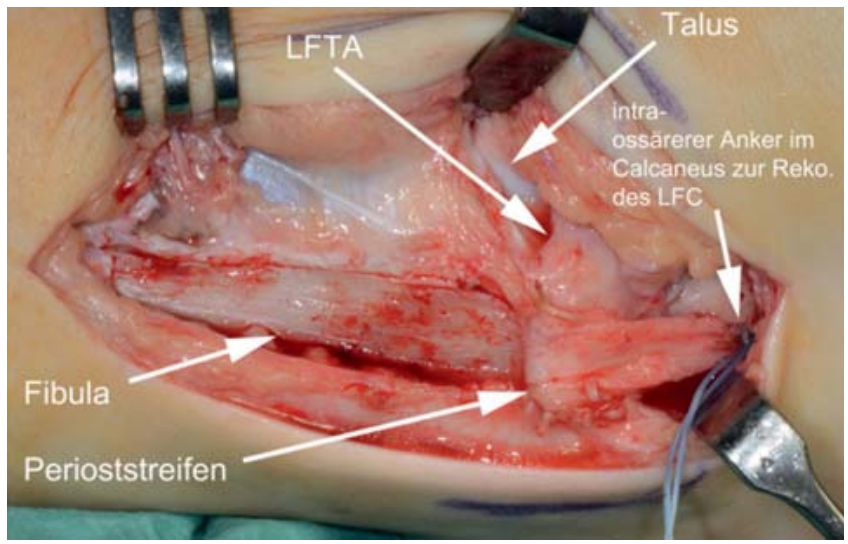

Abb. 16 Beispiel eines intraossär verankerten Perioststreifens zur Rekonstruktion des LFC in einzügeliger Technik bei suffizientem LFTA.
Größe des Entnahmevolumens eine wesentliche Rolle spielte.

\section{Instabilität}

Die Sprunggelenksdistorsion führt neben den möglichen erwähnten intraartikulären Verletzungsfolgen zu ligamentären Verletzungen. Diesbezüglich sollten Verletzungen der Syndesmose, des fibularen (äußeren) und medialen Bandapparats differenziert werden. Am häufigsten ist das sog. Supinationstrauma bestehend aus einer Kombination von Inversion, Adduktion und Plantarflexion des Fußes. Auf diese Verletzungsentität des fibularen Bandapparats soll an dieser Stelle eingegangen werden.

Die fibulare Bandläsion sollte systematisch in Grade klassifiziert werden [10]: Grad-I-Läsionen sind Distorsionen ohne wesentliche strukturelle Rupturen im Sinne der Bänderdehnung. Grad-II-Läsionen werden charakterisiert durch Partialrupturen des fibularen Bandapparats mit diskreter bis mäßiger Instabilität. Grad-III-Läsionen schließen Komplettrupturen ein, die zu relevanten Instabilitäten führen. Anatomisch sind von ventral nach dorsal das Lig. fibulotalare anterius (LFTA), das Lig. fibulocalcaneare (LFC) und das Lig. fibulotalare posterius (LFTP) zu differenzieren. Die Verletzungshäufigkeit ist in der gleichen Reihenfolge anzugeben, wobei Verletzungen des Lig. fibulotalare posterius eine Rarität darstellen. Biologisch können 3 Stadien der ligamentären Heilung differenziert werden [10]: 1. inflammatorische Phase (bis 10 Tage nach dem Trauma); 2. Proliferationsphase 4-8 Wochen nach dem Trauma; 3. Remodeling (bis zu 1 Jahr nach dem Trauma). Interindividuelle Unterschiede können bez. der
Dauer der einzelnen Phasen bestehen. Für die Akutbehandlung werden die operative und konservative Therapie kontrovers diskutiert. Weitere Fragen werfen in der konservativen Therapie die Immobilisierung und die funktionelle Nachbehandlung auf sowie die Art der effektivsten externen Ruhigstellung [10].

Werden die vergleichenden Studien (operative vs. konservative Therapie) zusammengefasst, zeigt sich für die operative Therapie bzw. Naht ein Vorteil hinsichtlich Stabilität und Rezidivverletzung gegenüber der konservativen Therapie. Allerdings müssen die Nachteile einer höheren Komplikationsrate nach operativer Rekonstruktion diesen Vorteilen gegenübergestellt werden, sodass für die Mehrheit der fibularen Bandläsionen die konservative Therapie empfohlen werden kann. Die Operation hat insbesondere für Leistungssportler mit hohem Stabilitätsanspruch nach wie vor ihren Stellenwert nicht verloren.

Die Analysen zur Frage der konservativen Therapie sind in ihrer Evidenz nach wie vor beeinträchtigt, wobei sich Hinweise zeigen, dass eine kurzfristige Ruhigstellung von 10 Tagen nach Trauma in einem Unterschenkelgips (Cast bzw. Äquivalent) für die inflammatorische Phase gefolgt von einer funktionellen Nachbehandlung mit einem semirigiden Brace bzw. Cast (beispielsweise Vacoankle ${ }^{\circledR}$, Aircast ${ }^{\circledR}$ etc.) günstige Ergebnisse bringt. Für die Akut-Therapie und die darauffolgenden 5 Tage sollte die PECH-Regel (Pause, Eis bzw. Kühlung, Compression, Hochlagerung) zur Reduktion der akuten Schwellung und Einblutung Beachtung finden.
Schnürbandagen zeigten bez. der Stabilität bessere kurzfristige Ergebnisse als normale Bandagen. Der Tape-Verband im Vergleich zum semirigiden Brace zeigte vergleichbare Ergebnisse bez. Schmerz und funktionellem Outcome, wobei deutlich niedrigere Komplikationsraten der Haut mit dem semirigiden Brace auftraten. Werden diese Ergebnisse zusammengefasst, kann man für Grad-III-Läsionen eine kurzfristige Ruhigstellung von längstens 10 Tagen gefolgt von einer Stabilisierung mit einer semirigiden Orthese empfehlen. Für alle anderen Läsionen kann in Abhängigkeit vom Zustand der Weichteile gleich der semirigide Brace eingesetzt werden.

Kommt es im mittel- bis langfristigen Verlauf trotzdem zu einer Beschwerdesymptomatik mit mehr oder weniger bestehender Instabilität, so hat die Arthroskopie, wie oben beschrieben, einen wichtigen Stellenwert. Besteht jedoch zusätzlich eine Instabilitätssymptomatik, so sollte die Diagnostik relevante laterale tibiotalare Aufklappbarkeiten ausschließen. Neuromuskuläres Propriozeptionstraining zeigte einen Effekt, sodass dies bei chronischer Instabiltätssymptomatik zunächst erfolgen sollte. Die Arthroskopie des OSG hilft, neben den gehaltenen Aufnahmen (a.-p. und seitlich), zu differenzieren, ob das LFTA allein, das LFTA und das LFC oder in besonderen Fällen das LFC isoliert adressiert werden muss [11]. Ein Beispiel einer isolierten LFC-Insuffizienz, die arthroskopisch gesichert und in gleicher Sitzung mit einer einzügeligen Periostlappenplastik transossär stabilisiert wurde, ist Abb.14 zu entnehmen. Die Stabilisierungspflichtigkeit des LFTP ist eine Rarität. 
Eine relativ neue Behandlungsalternative stellt die periartikuläre Injektion von Hyaluronsäure (HA) dar. Petrella et al. konnten in einer prospektiv randomisierten Doppelblindstudie einen signifikant positiven Effekt von periartikulär injizierter HA im Bereich des LFTA und LFC gegenüber der Placeboinjektion mit identischer Physiotherapie bzw. Schienenbehandlung objektivieren [12]. Es tritt signifikant schneller Schmerzfreiheit und deutlich früher Sportfähigkeit ein. Auch nach 2 Jahren ist die Inzidenz rezidivierender Distorsionen (bzw. residuelle Instabilität) niedriger nach HA-Injektion. Das biologische Prinzip besteht aus einer schnelleren und stabileren Quervernetzung von Fibrin und Kollagen mit und durch HA. Dieser biologische „interne Wundverband“ nimmt somit eine Zwischenstellung zwischen der operativen Naht und der rein konservativen Behandlung der Außenbandruptur ein.

\section{Schlussfolgerungen}

Die Arthroskopie des OSG hat sich als diagnostische und therapeutische Option etabliert und durchgesetzt. Die Einsatzmöglichkeiten beinhalten in der akuten Behandlung die Bergung frischer osteochondraler Flakes mit stadiengerechter Behandlung des Knorpelschadens. In der subakuten und chronischen Situation stellt die Arthroskopie gerade bei persistierenden Beschwerden in der Behandlung des knöchernen und weichteiligen Impingements eine wichtige Behandlungssäule dar, insbesondere dann, wenn die konservativen Behandlungsmaßnahmen keinen befriedigenden Effekt erbracht haben. Die Entfernung posttraumtischer intraartikulärer Briden bis hin zum Meniskoid ist arthroskopisch sicher und reproduzierbar möglich. Knöchern bedingtes Impingement nach knöchernen Ausrissen, Ossikelbildung, Ossifikationen sowie freie Gelenkkörper sollten primär arthroskopisch entfernt werden. Neben dem Staging des OSG sollte und kann die tibiotalare Stabilität arthroskopisch selektiv geprüft werden. Die Behandlung von osteochondralen Läsionen muss stadiengerecht erfolgen im Rahmen des arthroskopischen Stagings, wobei die Mikrofrakturierung insbesondere für kleinere Knorpelläsionen $\left(<1,5 \mathrm{~cm}^{2}\right)$ empfohlen wird. Die Langzeitprognose ist derzeit nur mit Einschränkungen beurteilbar. Für große isolierte Knorpelläsionen oder bei koinzidenten subchondralen Knochendefekten sind entweder die OCT oder die kortikospongiöse Transplantation mit MACT möglich. Auch hier sind die Langzeiterfahrungen begrenzt und müssen durch Studien überprüft werden. Das dorsale arthroskopishe Vorgehen hat sich bei dorsalem Impingement auch bewährt, wobei man jedoch eine höhere neurologische Komplikationsrate beachten muss.

\section{Literatur}

${ }^{1}$ van Dijck CN, Tol JN, Verheyen CC et al. A prospective study of prognostic factors concerning the outcome of arthroscopic surgery for anterior ankle impingement. Am J Sports Med 1997; 25: 737-745

2 Nickisch F, Barg A, Saltzman Cet al. Postoperative complications of posterior ankle and hindfoot arthroscopy. J Bone Joint Surg Am 2012; 94: 439-446

3 Phisitkul P, Tennant J, Amendola A. Is there any value to arthroscopic debridement of ankle osteoarthritis and impingement? Foot Ankle Clin N Am 2013; 18: 449-458

${ }^{4}$ Murawski CD, Kennedy JG. Operative treatment of osteochondral lesions of the talus. J Bone Joint Surg Am 2013; 95: 1045-1054

5 Chuckpaiwong B, Berkson EM, Theodore GH. Microfracture for osteochondral lesions of the ankle: outcome analysis and outcome predictors of 105 cases. Arthroscopy 2008; 24: 106-112

${ }^{6}$ Kennedy JG, Murawski CD. The treatment of osteochondral lesions of the talus with autologous osteochondral transplantation and bone marrow aspirate concentrate: surgical technique. Cartilage 2011; 2: 327-336

7 Shapiro F, Koide S, Glimcher MJ. Cell origin and differentiation in the repair of full-thickness defects of articular cartilage. J Bone Joint Surg Am 1993: 75: 532-553

${ }^{8}$ Lamb J, Murawski CD, Deyer TW et al. Chevron-type medial malleolar osteotomy: a functional, radiographic and quantitative T2mapping MRI analysis. Knee Surg Sports Traumatol Arthrosc 2013; 21: 1283-1288

${ }_{9}^{9}$ Paul J, Sagstetter A, Kriner M et al. Donor-site morbidity after osteochondral autologous transplantation for lesions of the talus. J Bone Joint Surg Am 2009; 91: 1683-1688

10 Petersen W, Rembitzki IV, Koppenburg AG et al. Treatment of acute ankle ligament injuries: a systematic review. Arch Orthop Trauma Surg 2013; 133: 1129-1141

${ }^{11}$ Kerr HL, Bayley E, Jackson R et al. The role of arthroscopy in the treatment of functional instability of the ankle. Foot Ankle Surg 2013; 19: 273-275

12 Petrella MJ, Cogliano A, Petrella RJ. Long-term efficacy and safety of periarticular hyaluronic acid in acute ankle sprain. Phys Sportsmed 2009; 37: 64-70

\section{Dr. med. Atesch Ateschrang \\ Leitender Arzt der Sektion \\ Sporttraumatologie und arthroskopische Chirurgie Dr. med. Steffen Schröter Oberarzt der Sektion Sporttraumatologie und arthroskopische Chirurgie Prof. Dr. med. Ulrich Stöckle} Ärztlicher Direktor

Berufsgenossenschaftliche Unfallklinik Tübingen Eberhard-Karls-Universität Tübingen Schnarrenbergstraße 95 72076 Tübingen aateschrang@bgu-tuebingen.de 